\title{
Towards String Support in JayHorn (Competition Contribution)
}

\author{
Ali Shamakhi ${ }^{1} \mathbb{D}(\bowtie)$, Hossein Hojjat $^{1,2} \mathbb{D}$, and Philipp Rümmer ${ }^{3} \mathbb{D}$ \\ 1 University of Tehran, Tehran, Iran \\ \{ali.shamakhi,hojjat\}@ut.ac.ir \\ 2 Tehran Institute for Advanced Studies, Tehran, Iran \\ 3 Uppsala University, Uppsala, Sweden \\ philipp.ruemmer@it.uu.se
}

\begin{abstract}
JayHorn is a Horn clause-based model checker for Java programs that has been competing at SV-COMP since 2019. An ongoing research and implementation effort is to add support for String data-type to JayHorn. Since current Horn solvers do not support strings natively, we consider a representation of (unbounded) strings using algebraic datatypes, more precisely as lists. This paper discusses Horn clause encodings of different string operations, and presents preliminary results.
\end{abstract}

\section{The JayHorn Approach and Architecture}

We start by summarising the approach used in JayHorn, and refer to earlier papers $[5,6,7]$ for more details. JayHorn is a verification tool that encodes sequential Java programs as sets of Constrained Horn Clauses (CHCs) in order to check for possible assertion violations. The main $\mathrm{CHC}$ encoding in JayHorn is inspired by refinement types [2] and liquid types [8], and characterises programs in terms of method contracts, state invariants, and instance invariants of classes [5]. This encoding is over-approximate, and can prove absence of assertion violations. In order to find counterexamples, i.e., prove existence of violations, JayHorn also offers a bounded, under-approximate program encoding.

JayHorn is entirely implemented in Java, and uses the Soot framework [10] to process Java bytecode, and the CHC solver Eldarica [3] to solve Horn clauses.

\section{Encoding of String Operations}

In this paper, we focus on the handling of Strings and their operations, a feature of Java that was not previously supported by JayHorn. Since JayHorn verifies programs without imposing bounds on the number of execution steps or the size of input data, our goal is to handle also unbounded strings. Unfortunately, while there has been significant progress in SMT solving for strings, current CHC solvers do not yet support strings natively. We therefore use recursive algebraic data types to model strings, and follow the approach proposed in [4]: strings are represented using lists, with a binary constructor cons and the constant nil.

(C) The Author(s) 2021

J. F. Groote and K. G. Larsen (Eds.): TACAS 2021, LNCS 12652, pp. 443-447, 2021.

https://doi.org/10.1007/978-3-030-72013-1_29 
There are two ways to encode a string using cons and nil. The Left-To-Right (LTR) encoding starts with the leftmost character of the string. For example, "Jay" = cons('J', cons('a', cons('y', nil))). The Right-to-Left (RTL) encoding starts with the rightmost character. Each encoding has its own benefits and drawbacks in modeling various operations, an aspect we evaluate in this paper.

Three different LTR encodings of the concatenation operation are described in [4], and equivalent RTL encodings are easy to define. Moving beyond concatenation, in this paper we show models of some of the more involved operations.

\subsection{The CompareTo Operation}

The String. compareTo method in Java returns an integer, which is the difference of the length of strings if one of the strings is a prefix of the other (e.g., "cat".compareTo("c") == 2), or the difference of their leftmost same-index different characters otherwise (e.g., "card". compareTo ("cash") == -1, since their leftmost same-index different characters are ' $r$ ' and ' $s$ ', respectively).

The method is modeled using predicate $P_{\text {rec }}$ (left, right, comparison_result) under LTR encoding, which allows us to recursively remove leftmost characters from both strings to reach a state which the comparison_result is known.

$$
\begin{aligned}
P_{r e c}(x, \text { nil, len }(x)) & \leftarrow \text { true } \\
P_{\text {rec }}(\text { nil, } y,-\operatorname{len}(y)) & \leftarrow \text { true } \\
P_{r e c}(x, x, 0) & \leftarrow \text { true } \\
P_{r e c}(\operatorname{cons}(j, x), \operatorname{cons}(k, y), j-k) & \leftarrow j \neq k \\
P_{r e c}(\operatorname{cons}(h, x), \operatorname{cons}(h, y), d) & \leftarrow P_{r e c}(x, y, d)
\end{aligned}
$$

The predicate under RTL encoding needs an extra argument to keep track of whether the comparison_result is based on character difference or not, so the predicate is $P_{r e c}^{\prime}(l e f t$, right, comparison_result, char_diff). The clauses use the len function to compute the length of a string, which is a built-in function in Eldarica.

$$
\begin{aligned}
P_{r e c}^{\prime}(x, \text { nil, len }(x), \text { false }) & \leftarrow \text { true } \\
P_{r e c}^{\prime}(\text { nil, } y,- \text { len }(y), \text { false }) & \leftarrow \text { true } \\
P_{r e c}^{\prime}(x, x, 0, \text { false }) & \leftarrow \text { true } \\
P_{r e c}^{\prime}(\operatorname{cons}(h, x), y, d+1, \text { false }) & \leftarrow P_{r e c}^{\prime}(x, y, d, \text { false }) \wedge \operatorname{len}(x) \geq \operatorname{len}(y) \\
P_{r e c}^{\prime}(x, \operatorname{cons}(h, y), d-1, \text { false }) & \leftarrow P_{r e c}^{\prime}(x, y, d, \text { false }) \wedge \operatorname{len}(x) \leq \operatorname{len}(y) \\
P_{r e c}^{\prime}(\operatorname{cons}(j, x), \operatorname{cons}(k, x), j-k, \text { true }) & \leftarrow j \neq k \\
P_{r e c}^{\prime}(\operatorname{cons}(h, x), y, d, \text { true }) & \leftarrow P_{r e c}^{\prime}(x, y, d, \text { true }) \\
P_{r e c}^{\prime}(x, \operatorname{cons}(h, y), d, \text { true }) & \leftarrow P_{r e c}^{\prime}(x, y, d, \text { true })
\end{aligned}
$$

\subsection{Integer to String conversion}

The integer to string conversion relies on extracting digits one by one, which is done using integer arithmetic. Under LTR encoding, during the conversion process, the pre-condition stores the rest of the input after removing the converted digits so far starting from the lowest position. For example, if the number is 
$i=\overline{d_{n-1} \cdots d_{0}}$ and the converted string so far is $s=" d_{k-1} \cdots d_{0} "$, the rest of the number will be $r=\overline{d_{n-1} \cdots d_{k}}$ which is stored at the pre-condition.

The pre-condition in RTL encoding stores the offset of the next digit that needs to be extracted, since extracting digits from highest place values requires knowing their positions.

\section{$2.3 \quad$ StartsWith and EndsWith}

The encoding of String.startsWith method needs to consider different states of both strings and their relation, which leads to multiple recursive relations.

For example, if $x$ starts with $y$, we can prepend $c$ to both strings under LTR encoding (to get $x^{\prime}$ and $y^{\prime}$ ) and the condition holds on the resulting strings (i.e. $x^{\prime}$ starts with $y^{\prime}$ ). For another example, if $x$ does not start with $y$ and len $(x) \geq \operatorname{len}(y)$ we can append $c$ to $x$ under RTL encoding (to get $x^{\prime}$ ) and the condition holds on the resulting string (i.e. $x^{\prime}$ does not start with $y$ ).

$S_{\text {rec }}(\operatorname{cons}(h, x), \operatorname{cons}(h, y)$, true $) \leftarrow S_{\text {rec }}(x, y$, true $)$

(LTR) $S_{\text {rec }}(\operatorname{cons}(j, x), \operatorname{cons}(k, y)$, false $) \leftarrow j \neq k$

(RTL) $\quad S_{\text {rec }}(\operatorname{cons}(h, x), y$, true $) \leftarrow S_{\text {rec }}(x, y$, true $)$

(RTL) $S_{\text {rec }}(\operatorname{cons}(j, x), \operatorname{cons}(k, x)$, false $) \leftarrow j \neq k$

(RTL) $\quad S_{\text {rec }}(\operatorname{cons}(h, x), y$, false $) \leftarrow S_{\text {rec }}(x, y$, false $) \wedge \operatorname{len}(x) \geq \operatorname{len}(y)$

(RTL) $\quad S_{\text {rec }}(x$, cons $(h, y)$, false $) \leftarrow S_{\text {rec }}(x, y$, false $)$

The RTL encoding of endsWith is the same as LTR encoding of startsWith, and the LTR encoding of endsWith is the same as RTL encoding of startsWith.

\subsection{CharAt}

The encoding definition of String.charAt relies on the fact that prepending a character to a string under LTR encoding increases indices of all previous characters by one, while appending a character to a string under RTL encoding does not change those indices.

$\begin{array}{rrrl}(\mathrm{LTR}) & C h A t_{\text {rec }}(\operatorname{cons}(h, t), 0, h) & \leftarrow \operatorname{true} \\ (\mathrm{LTR}) & C h A t_{r e c}(\operatorname{cons}(h, t), i+1, c) & \leftarrow C h A t_{r e c}(t, i, c) \wedge 0 \leq i<\operatorname{len}(t) \\ (\mathrm{RTL}) & C h A t_{r e c}(\operatorname{cons}(h, t), \operatorname{len}(t), h) & \leftarrow \operatorname{true} \\ (\mathrm{RTL}) & C h A t_{r e c}(\operatorname{cons}(h, t), i, c) & \leftarrow C h A t_{r e c}(t, i, c) \wedge 0 \leq i<\operatorname{len}(t)\end{array}$

\section{Performance of the String Encoding}

The following table shows the results of JayHorn on the 53 problems in the SVCOMP Java track that involve strings. Many of the programs contain string 
operations that are not yet handled in JayHorn, but the results already make it possible to compare encoding choices. Uniformly, RTL performs better than LTR (probably because appending characters to strings is more common than adding characters in the beginning), and the under-approximating CHC encoding of JayHorn performs better than the over-approximate encoding (probably because over-approximation too often loses information about string contents). The choice between Iterative, Recursive, or Recursive-with-precondition [4] for string concatenation surprisingly had no effect on the results.

\begin{tabular}{|c|c|c|c|c|c|c|c|c|c|c|c|c|}
\hline \multirow{2}{*}{$\begin{array}{c}\text { Encoding } \\
\text { Choices }\end{array}$} & \multicolumn{4}{|c|}{ Iterative } & \multicolumn{4}{c|}{ Recursive } & \multicolumn{2}{c|}{ RecursiveWithPrec } \\
\cline { 2 - 14 } & U-Approx & O-Approx & U-Approx & O-Approx & U-Approx & O-Approx \\
\cline { 2 - 13 } & LTR & RTL & LTR & RTL & LTR & RTL & LTR & RTL & LTR & RTL & LTR & RTL \\
\hline \# Solved & 4 & 6 & 1 & 3 & 4 & 6 & 1 & 3 & 4 & 6 & 1 & 3 \\
\hline Avg. Time (s) & 81 & 79 & 7.5 & 16 & 79 & 78 & 7.6 & 16 & 77 & 78 & 7.7 & 16 \\
\hline
\end{tabular}

In other respects, JayHorn performed similarly in SV-COMP 2021 [1] as in the two previous years. JayHorn gave one incorrect answer, for the problem UnsatAddition02 and due to the use of unbounded integer arithmetic instead of correct Java machine arithmetic semantics. JayHorn could correctly prove 125 benchmarks safe, and 151 benchmarks unsafe. Changes compared to 2020 include 59 of the 64 MinePump benchmarks (by encoding enums, see Section 4) and 6 of the 53 string benchmarks that JayHorn solves now.

The biggest factor influencing the performance of JayHorn in SV-COMP is still the incomplete model of the Java API in JayHorn, given the large number of API tests among the SV-COMP Java benchmarks. Our work on supporting Strings, described in this paper, is one of the efforts to address the situation.

\section{Tool Setup}

The version submitted to SV-COMP 2021 is JayHorn version 0.7.5-strings, ${ }^{4}$ which is also available on Zenodo [9]. In the configuration used in the competition, ${ }^{5}$ JayHorn only applies the Horn solver Eldarica. The Benchexec tool info module is called jayhorn.py and the benchmark definition file jayhorn.xml. JayHorn competes in the Java category.

Since JayHorn only has incomplete support for Java enums, in this year we added a small source transformation tool $^{6}$ to JayHorn that has the purpose of replacing enums with simple integer variables. The script used in the competition applies the transformation tool to the benchmark source code prior to compilation to bytecode.

\footnotetext{
${ }^{4}$ https://github.com/jayhorn/jayhorn/releases/tag/v0.7.5-strings

5 Java options: $-\mathrm{Xss} 40000 \mathrm{k}-\mathrm{Xmx} 12 \mathrm{~g}$

JayHorn options: -inline-size 50 -conservative -specs -string-encoding recursiveWithPrec -string-direction rtl

${ }^{6}$ https://github.com/jayhorn/jayhorn/tree/devel/enum-eliminator
} 


\section{Software Project and Contributors}

JayHorn was initially developed by Temesghen Kahsai, Philipp Rümmer, and Martin Schäf, with contributions by Daniel Dietsch, Rody Kersten, Huascar Sanchez, and Valentin Wüstholz [6,7]. Further development of the tool is at the moment mainly carried out by the authors of this paper. JayHorn is open source, and distributed under MIT license on https://github.com/jayhorn/jayhorn.

Acknowledgements. The work on JayHorn has been supported by the Swedish Research Council (VR) under grant 2018-04727, by the Swedish Foundation for Strategic Research (SSF) under the project WebSec (Ref. RIT17-0011), and by grants from Microsoft and Amazon Web Services.

\section{References}

1. D. Beyer. Software verification: 10th comparative evaluation (SV-COMP 2021). In Proc. TACAS (2), LNCS 12652. Springer, 2021.

2. T. Freeman and F. Pfenning. Refinement types for ML. In PLDI, pages 268-277, New York, NY, USA, 1991. ACM.

3. H. Hojjat and P. Rümmer. The ELDARICA Horn solver. In FMCAD. IEEE, 2018.

4. H. Hojjat, P. Rümmer, and A. Shamakhi. On strings in software model checking. In APLAS. Springer, 2019.

5. T. Kahsai, R. Kersten, P. Rümmer, and M. Schäf. Quantified heap invariants for object-oriented programs. In LPAR. EasyChair, 2017.

6. T. Kahsai, P. Rümmer, H. Sanchez, and M. Schäf. JayHorn: A framework for verifying Java programs. In $C A V$. Springer, 2016.

7. T. Kahsai, P. Rümmer, and M. Schäf. JayHorn: A Java model checker - (competition contribution). In D. Beyer, M. Huisman, F. Kordon, and B. Steffen, editors, TACAS: TOOLympics, volume 11429 of LNCS, pages 214-218. Springer, 2019.

8. P. M. Rondon, M. Kawaguchi, and R. Jhala. Liquid types. In R. Gupta and S. P. Amarasinghe, editors, PLDI, pages 159-169. ACM, 2008.

9. A. Shamakhi, H. Hojjat, and P. Rümmer. JayHorn artifact at SV-COMP 2021. Zenodo: https://doi.org/10.5281/zenodo.4485702.

10. R. Vallée-Rai, L. Hendren, V. Sundaresan, P. Lam, E. Gagnon, and P. Co. Soot a Java Optimization Framework. In CASCON, 1999.

Open Access This chapter is licensed under the terms of the Creative Commons Attribution 4.0 International License (https://creativecommons.org/licenses/by/4.0/), which permits use, sharing, adaptation, distribution and reproduction in any medium or format, as long as you give appropriate credit to the original author(s) and the source, provide a link to the Creative Commons license and indicate if changes were made.

The images or other third party material in this chapter are included in the chapter's Creative Commons license, unless indicated otherwise in a credit line to the material. If material is not included in the chapter's Creative Commons license and your intended use is not permitted by statutory regulation or exceeds the permitted use, you will need to obtain permission directly from the copyright holder.

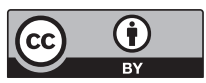

